\title{
Sciatic Nerve Neuropathy in Cynomolgus Monkey Macaca Fascicularis: Altered Leg Usage and Peripheral Nerve Firing
}

Ning Guo ${ }^{1,2}$, Xiyao $\mathrm{Gu}^{3}$, Yikuan Xie ${ }^{4}$, Jun Zhao ${ }^{3}$, Qinglian Xie ${ }^{5}$, Guoping Zhao ${ }^{5}$, Meilei Jin ${ }^{5}$, Zhiqi Zhao ${ }^{3}$, Hong Zou ${ }^{5}$, Yuqiu Zhang ${ }^{3}$, Gang Jason Jin ${ }^{2}$, Lei Yu $6^{*}$

${ }^{1}$ Shanghai University of Traditional Chinese Medicine, Shanghai, China

${ }^{2}$ ShanghaiBio Corporation, 675 US Highway One, North Brunswick, New Jersey 08902, USA

3 Institute of Neurobiology, Institutes of Brain Science and State Key Laboratory of Medical Neurobiology, Fudan University, Shanghai, China

${ }^{4}$ Department of Physiology, Institute of Basic Medical Sciences, Chinese Academy of Medical Sciences, School of Basic Medicine, Peking Union Medical College, Beijing, China

${ }^{5}$ Shanghai Institutes for Biological Sciences, Chinese Academy of Sciences, Shanghai, China

${ }^{6}$ Department of Genetics \& Center of Alcohol Studies, Rutgers University, 607 Allison Road, Piscataway, New Jersey 08854, USA

*Corresponding author: Lei Yu, Department of Genetics \& Center of Alcohol Studies, Rutgers University, 607 Allison Road, Piscataway, New Jersey 08854, USA; Tel.: +1-848-445-0794, fax: +1-732-445-3500, E-mail: yu@biology.rutgers.edu

Received date: Sep 25, 2014, Accepted date: Nov 18, 2014, Published date: Nov 22, 2014

Copyright: ( 2014 Guo N, et al. This is an open-access article distributed under the terms of the Creative Commons Attribution License, which permits unrestricted use, distribution, and reproduction in any medium, provided the original author and source are credited.

\begin{abstract}
Sciatic nerve is susceptible to trauma and injuries. Animal models for sciatic nerve trauma are mostly in rodents, with limited information about injury-induced neuropathy in non-human primates. We constructed a model of sciatic nerve neuropathy (SNN) in the cynomolgus macaque monkey, Macaca fascicularis, with mild injury to, but without transection of, the sciatic nerve. Monkeys' behavioral and physiological properties were characterized. SNN led to reduced leg usage as well as muscle atrophy. Sciatic nerve retained the ability of nerve signal transduction, and showed a flat-line type of firing rate profile, consistent with the hypothesis of injury-resulted hyper-sensitization. These data suggest that mild injury to sciatic nerve result in long-lasting malfunction and neuropathy in monkeys. This model may serve as a non-human primate model to study functional changes, as well as underlying pathological mechanisms, of traumatic injury to the sciatic nerve.
\end{abstract}

Keywords: Sciatic nerve; Neuropathy; Muscle atrophy; Multiple-unit recording; Non-human primates

\section{Introduction}

Sciatic nerve, the longest nerve in the body, runs from the lower spine through the buttock and hips, then down the back of the leg. The long course and the anatomical position of the sciatic nerve make it susceptible to injuries of various causes, such as infections, metabolic, chemical, or traumatic insults. Traumas that range from simple nerve fiber compression to complete transection are common, resulting in motor, sensory, autonomic mal-functions, as well as reduced muscle recruitment in the segment of the body that the nerve innervates [1-8]. Pain and palsy are two major aspects of the sensory disturbances associated with sciatic nerve neuropathy.

Animal models, employing total transection or partial damage to the sciatic nerve and its branches, have been developed to study the underlying mechanisms of the progression and manifestation of sciatic nerve injury. Most efforts were made in rodents. Complete transection of the sciatic nerve produced severe autotomy behavior towards the anesthetic limb in rats and mice [9]. Allodynia and hyperalgesia are commonly observed in rodent models employing partial sciatic nerve damage, such as chronic constriction injury $[10,11]$, partial nerve ligation $[12,13]$, segmental spinal nerve ligation $[14,15]$, and spared nerve injury $[16,17]$.

Non-human primates are closely related to human, and possess nervous systems that are similar to those in human, making them desirable model organisms to study neuropathy-related biological phenomena and pathological mechanisms. However, nerve injury studies in non-human primates are limited.

In one report, the authors used a macaque monkey, Macaca mulatta as the experimental animal, and generated a sciatic nerve injury model by transecting the spinal nerves (L5, L5\&L6, L5\&L6\&L7) that contribute to the sciatic nerve [18]. The affected legs of L5\&L6\&L7transected monkeys were severely paretic and disabled with muscle atrophy in the plantar extensors and dorsiflexors [18]. The L5- and L5\&L6-transected monkeys demonstrated moderate paresis and slight functional disability in affected legs. Muscle atrophy was also limited [18]. L7 spinal nerve ligation model was developed in another macaque monkey, Macaca fascicularis. Behavior tests showed a bilateral mechanical allodynia in monkeys with L7 spinal nerve ligation, whereas the ipsilateral side showed stronger sensitization $[19,20]$; no sciatic nerve palsy or muscle atrophy was reported.

In the present study, we used Macaca fascicularis, commonly known as the crab-eating macaque, long-tailed macaque, or cynomolgus monkey. We established an injury model of sciatic nerve neuropathy (SNN), with the intent for a mild type of injury that does not involve nerve transection, so that nerve transduction can still take place. We reasoned that under such mild injury conditions, behavioral and physiological outcomes may more closely model the chronic, long-term nature of sciatic nerve neuropathy. Here we report the initial characterization of the behavioral and electrophysiological effect of SNN. 


\section{Materials and Methods}

This study adhered to the principles of RIGOR guidelines [21,22], including blinding of the study, randomization of treatment groups, the use of control groups, and the usage of statistical analysis.

\section{Animals}

Eighteen male monkeys (Macaca fascicularis) at the age of 3 years were used in the study. All animals were supplied by, and experiments were performed in, the laboratories at Hainan Jingang Laboratory Animal Co. Ltd., an AAALAC-accredited facility located in Hainan Province in southern China. The local climate in Hainan Province is similar to that of the natural habitat of these monkeys. All experiment protocols were approved by the Institutional Animal Care and Use Committee (IACUC) at Hainan Jingang Laboratory Animal Co. Ltd., adhered to the guidelines of the Committee for Research and Ethical Issues of IASP, and were consistent with the National Institutes of Health Guide for the Care and Use of Laboratory Animals. Before experiments, monkeys were transferred from group housing to individual cages $(80 \times 60 \times 70 \mathrm{~cm}, \mathrm{LxWxH})$. There were two rows of cages facing each other in the laboratory, with solid dividers between neighboring cages; thus, the monkeys could not see their immediate neighbors, but had visual contact with the monkeys in the opposite row in the room. Animals also could reach out of the cage and had limb contact with the neighboring monkeys. The room was well ventilated. Room lighting was supplied mainly by natural light, with some fluorescent lighting to facilitate night-time video recording of behavior. Monkeys were served three meals a day with regular monkey chow, plus seasonal fruits in the afternoon. Drinking water was provided ad libitum. Meal service, room cleaning, and animal care were performed by the technicians at Hainan Jingang Laboratory Animal Co. Ltd., with the supervision of certified veterinarians.

\section{Sciatic nerve neuropathy (SNN) model}

Anesthesia: The monkey cages were designed with a back plate that could be pulled toward the front of the cage, so that the monkey could be restrained. Monkeys were first sedated in the cage with $5 \mathrm{mg} / \mathrm{kg}$ i.m. ketamine, and were subsequently anesthetized with i.p. injection of $2 \%$ pentobarbital sodium at the dosage of $30 \mathrm{mg} / \mathrm{kg}$. Additional pentobarbital solution was given as supplementary doses at $10 \mathrm{mg} / \mathrm{kg}$ when needed. The level of anesthesia was monitored by the reflex of hind leg to mechanical pinch. The ketamine and pentobarbital sodium dosages were based on those reported in the literature [23-25].

Surgery: The hair on the upper thigh area was removed by shaving. Using aseptic technique, an incision on the skin was made at the upper thigh level, and the common sciatic nerve about $20 \mathrm{~mm}$ in length was exposed after blunt dissection of connective tissues and through biceps femoris. Using 3-0 gut suture, four ligatures were made around the sciatic nerve sequentially from proximal to distal with about $3 \mathrm{~mm}$ distance between neighboring ligatures. Each ligature was gradually tightened until either the ligature formed a constricting groove on the sciatic nerve or a subtle muscle twitch was observed in the lower leg or the digits of the foot. The surgical site was closed in layers, and the skin cut was closed with silk suture. Penicillin G (200,000 U) was administered after the surgery.

Recovery: After the surgery, the monkey was returned to its home cage. A cloth blanket was wrapped around the monkey, and a heating fan was used to supply additional heating to help the monkey to recover from anesthesia. The monkey was frequently observed until it was awake and could move about in the cage.

\section{Video recording of monkey home cage activities}

A behavioral monitoring system was used to record monkeys' home cage activities throughout the duration of the study, consisting of video cameras, digital video recorders, and computers running video monitoring software. To minimize any blind area, for each monkey's cage, two video cameras were employed, one providing the top view and the other the front view of the entire cage.

\section{Analysis of leg usage during movement}

Video recording was reviewed offline for behavioral analysis. For leg usage analysis during animal's movement, video recording from 4 days after the sciatic nerve neuropathy surgery was used. For each monkey, 10 segments of video recording were randomly chosen and analyzed. Each video segment consisted of a 10-min recording, and the recording was viewed to find an incidence when the monkey moved about in the cage. During such movement, a 30 -second recording was used for scoring. If a leg was used during the movement, a score of " 1 " was given. If a leg was held up and not used, a score of "0" was given. The percentage of leg usage was calculated for each monkey from the 10 sampling video segments.

\section{Leg circumference measurement}

Pentobarbital anesthesia was as described above. After the animal was anesthetized, the leg circumferences at middle thigh level were measured on both legs of the animal. Heart beat and breathing rate were monitored. A heating pad was placed underneath the body of the animal. Body temperature was continuously monitored with a rectal temperature probe, and was kept at the physiological range with adjustment of the heating pad. Sciatic nerve and its three branches on both legs were surgically exposed. The skin was sutured around the rim of a stainless steel ring in order to form a funnel-shaped space, to be filled with pre-warmed mineral oil for keeping the nerve branches from dehydration.

\section{Electrophysiology recording}

The electric activities were recorded from a nerve bundle separated from the sural nerve, a branch of the sciatic nerve that innervates the shank area of the leg. Nerve activities of both legs were monitored with an oscilloscope, and were recorded with a MODEL $1700 \mathrm{AC}$ amplifier (A-M SYSTEMS) with different channels feeding into a DVD recorder (Sony DVD) via left or right sound channel.

To determine the receptive field of the nerve fibers under recording, a soft-paint brush with a pointed tip was used to brush confined spots in outer side of the shank area. The spot that produced the strongest electric signal as shown on the oscilloscope was marked as the receptive field of the nerve fibers under recording. Thereafter, spontaneous activity of the nerve fibers was recorded for $10 \mathrm{~min}$ before mechanical stimulation was applied.

Mechanical stimuli were applied to the receptive field using von Frey filaments. Different stimulus intensities were applied to the receptive field in successive steps from $1 \mathrm{~g}$ to $300 \mathrm{~g}$. Each stimulus lasted for approximately $5 \mathrm{sec}$, and the interval between two stimuli was 30 
sec. Euthanasia was performed after the completion of the electrophysiology recording.

Data acquisition and analyses: All recorded data were converted and transferred to computer via Powerlab 4/25 (AD Instruments), with a sampling rate for transferring data to computer at $10 \mathrm{KHz}$. The data were analyzed with Chart 5 for Windows (AD Instruments) using a plug-in software module named "spike histogram". To determine the noise threshold, 3 separate stretches during the spontaneous activity recording devoid of observable action potentials were selected. The highest basal potential in each piece was identified. The voltages of the 3 highest basal potentials were averaged, and the noise threshold was set as $120 \%$ of the average. The total number of action potentials during each stimulation period was acquired and was divided by the duration of the stimulus, and the result was designated as the "firing rate".

\section{Statistics}

Leg usage and circumference data are expressed as mean \pm SEM. Statistical analysis was conducted using unpaired t test with Welch's correction, with the significance level set at $\mathrm{p}<0.05$. The software package of GraphPad Prism was used for statistical analysis.

\section{Results}

\section{Sciatic nerve neuropathy ( $\mathrm{SNN}$ ) as a model of nerve injury}

We generated a nerve injury model of sciatic nerve neuropathy (SNN), using the non-human primate crab-eating macaque (Macaca fascicularis). The nerve injury technique was adapted from that first developed in rodents by Bennett and Xie [10]. Four ligatures were made around the sciatic nerve trunk with a $3-\mathrm{mm}$ space between adjacent ligatures. Each ligature was gradually tightened until either the ligature formed a constricting groove on the sciatic nerve or a subtle muscle twitch was observed in the lower leg or the digits of the foot. The intent was to create mild nerve trauma, so that neuropathy may develop. A total of nine monkeys received SNN surgery, with the side of the leg randomized (five received SNN on the left side leg, and four on the right side leg). Also, nine monkeys received sham surgery as controls, again with the side of the leg randomized.

\section{Effect of SNN on leg usage and muscle atrophy}

To determine the effect of sciatic nerve neuropathy, we analyzed monkey's behavioral pattern of leg usage during movement. Monkeys' behavioral pattern in the home cage is characterized by alternating staying stationary and moving about, so video recording from the fourth day after SNN or sham surgery was analyzed to quantify the extent of leg usage during movement. As shown in Figure 1, sham operated animals displayed equal preference for the two legs, with complete $(100 \%)$ usage of both legs during movement $(n=9)$, in the same manner as the equal preference for two legs before surgery (100\%). The SNN animals, on the contrary, heavily guarded the nerve injury leg, favoring greatly the contralateral hind leg during movement, and used the nerve injury leg only a minority of the time (Ipsilateral side: $26.7 \pm 10.3 \%$; contralateral side: $97.8 \pm 2.2 \%$; mean \pm $\mathrm{SEM}, \mathrm{n}=9$ for each group).

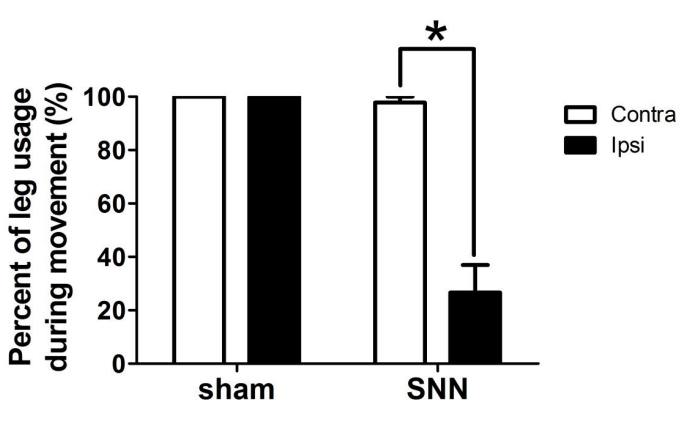

Figure 1: Percent of leg usage during movement. Leg usage during movement in the cage was scored 4 days after the sciatic nerve neuropathy surgery. For each animal, 10 sampling segments were performed, with 30 seconds in each segment. Normal movement with alternating usage of both legs was scored as normal, and skipping on one leg while keeping the other leg held-up in the air was scored as usage avoidance of the held-up leg. The 10 scores were averaged for each leg to obtain percent of leg usage. For each group of animals, the scores for leg of ipsilateral side and contralateral side were averaged separately, and data are presented as mean \pm SEM. Data of sham operated animals $(n=9)$ are plotted on the left, and data of SNN animals $(n=9)$ are plotted on the right. Filled bar, 'Ipsi' (ipsilateral side of either sham or SNN surgery); open bar, 'Contra' (contralateral side of either sham or SNN surgery). *, significantly different from contralateral side $(\mathrm{p}<0.05$, unpaired $t$ test with Welch's correction).

The reduced leg usage in SNN animals paralleled the appearance of muscle atrophy in the nerve injury leg. As seen in Figure 2, when the differential leg circumference at mid-thigh level was measured between the ipsilateral and the contralateral legs 2 months after the sham or SNN surgery, SNN animals showed a significant difference from the sham surgery animals (SNN: $14.5 \pm 1.9 \mathrm{~mm}$; sham: $1.9 \pm 1.1$ $\mathrm{mm}$; mean $\pm \mathrm{SEM}, \mathrm{n}=9$ for each group).

\section{Effect of SNN on electrophysiological properties of the sciatic nerve}

To examine the effect of SNN on the electrophysiological properties of the sciatic nerve, electrophysiological recording was carried out two months after SNN surgery. A nerve bundle was separated from the sural nerve, one of the three branches of sciatic nerve that innervates the shank area of the hind leg, and was subjected to electrophysiological recording. To determine the receptive field of the nerve fibers being recorded, various spots in the shank area were stimulated with a brush, and the spot that produced the strongest electric signal was marked as the representative of the receptive field of the nerve bundle being recorded. We first recorded the spontaneous activity of the nerve bundles. Subsequently, we recorded electrical responses to escalating mechanical stimulations delivered by von Frey filaments, ranging from $1 \mathrm{~g}$ to $300 \mathrm{~g}$, applied to the receptive field.

For each mechanical stimulation, "firing rate" was calculated by dividing the total number of action potentials by the duration of the stimulus. Since each nerve bundles contained different number of nerve fibers, the absolute values of the "firing rate" were not directly comparable with each other; therefore, the values for each nerve 
bundle were normalized to the data point at the maximum mechanical stimulus for that particular nerve bundle. Five animals were used in electrophysiological recording.

Bilateral recordings were obtained from all five animals (Figure 3). Two patterns of the electrophysiological responses to escalating stimulations were observed. For animals \#1 and \#2, the firing rate of nerve bundles on the contralateral side kept relatively flat when the force of the stimuli was low, and began to increase when the force exceeded $60 \mathrm{~g}$, showing response to the stimulation. In contrast, the firing rate of nerve bundles on the ipsilateral side reached plateau when the force was very low, and remained flat-lined as the force of stimuli increased. For animals \#3, \#4, and \#5, the firing rate of the sural nerve on the contralateral side fluctuated around the median level as the stimulation force increased.

\section{Discussion}

As the longest nerve in the body, sciatic nerve is susceptible to trauma and injuries. To study the effect on the sciatic nerve from such injuries in non-human primates, we constructed a model of sciatic nerve neuropathy (SNN) in the cynomolgus macaque monkey, Macaca fascicularis. Our intent was to establish a mild type of injury that does not involve nerve transection, so that nerve transduction can still take place, yet neuropathy may develop. We reasoned that under such mild injury conditions, behavioral and physiological outcomes may more closely model the chronic, long-term nature of sciatic nerve neuropathy. Our results demonstrated feasibility of the SNN model.

At the behavioral level, the SNN monkeys started to guard the nerve injury leg shortly (within a day or so) after the surgery, whereas the sham-surgery monkeys did not display any leg-guarding behavior. When the extent of leg usage during movement was quantified, the SNN monkeys preferentially utilized the contralateral hind leg during movement, and only used the nerve injury leg for a minority of the time (Figure 1). This was in contrast to complete bilateral usage of hind legs by the sham-operated animals (Figure 1). In an earlier study using Macaca Mulata as subjects, transection of spinal nerves that contributes to the sciatic nerve (L5\&L6\&L7) almost completely paralyzed the affected legs [18]. The affected legs were passively carried around with both hip and knee joint in flextion, which is very similar to what we observed in our study.

As would be expected from the reduced usage, the leg-guarding behavior in the SNN monkeys led to muscle atrophy in the nerve injury leg, resulting in significant difference in leg circumferences between the SNN leg vs. the contralateral side of the leg (Figure 2). Although there may be additional factors contributing to the muscle atrophy aside from the greatly reduced usage, our data do provide evidence in non-human primates for the adage "use it or lose it" in human rehabilitation medicine. Severe and progressive muscle atrophy of plantar extensors and dorsiflexsors was also observed in monkeys with L5\&L6\&L7 rhizotomy, which more reflected the effects of muscle denervation; while our data more indicated the functional relationship between muscle volume and daily usage.

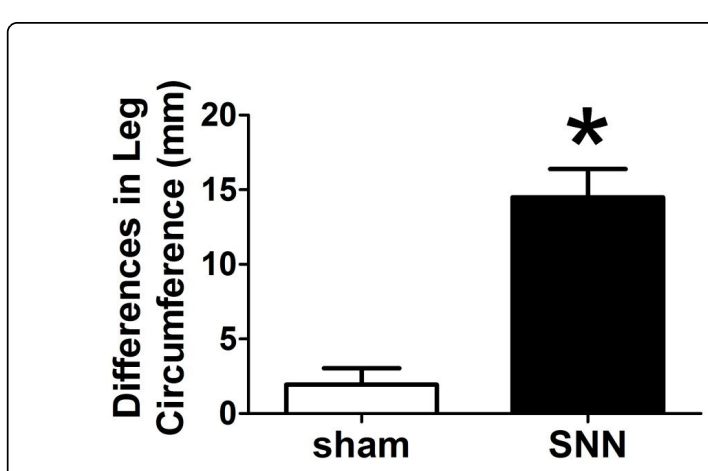

Figure 2: Differences in leg circumference. Circumference measurements of both legs were made at the mid-thigh level for each animal while under anesthesia. Measurement was undertaken by two different persons, and triplicate measurements were made for each leg in order to calculate an average. Difference in leg circumference score was derived by subtracting the circumference value of the ipsilateral side from that of the contralateral side. Data are presented as mean \pm SEM. Open bar, 'sham' (sham operated animals, $\mathrm{n}=9$ ); filled bar, 'SNN' (SNN operated animals, $\mathrm{n}=9$ ).*, significantly different from sham operated animals $(\mathrm{p}<0.05$, unpaired $t$ test with Welch's correction).

Electrophysiological recording showed that the SNN model did allow nerve transduction in the injured sciatic nerve, as we were able to record electric signals in these monkeys, both as spontaneous firing of the nerve at basal state, and upon mechanical stimulation of the receptive field for the nerve bundle being recorded. Two patterns of nerve firing were observed (Figure 3), both based on the firing rate profile of the contralateral side of the sciatic nerve. An orderly response pattern was seen for monkey $\# 1$ and $\# 2$, where the nerve firing rate began at the basal level, started to increase when the stimulation force exceeded $60 \mathrm{~g}$, and reached maximum at $300 \mathrm{~g}$ force. Monkeys \#3, \#4, and \#5 showed an irregular response pattern, with the nerve firing rate fluctuated around the median level as the stimulation force increased. It is not clear what contributed to the different response patterns of the normal sciatic nerve in these monkeys, but it is worth noting that, unlike experimental rodents from commercial lab animal suppliers, these monkeys appeared rather heterogeneous in their genetic origins. Thus, the two different response patterns of the sciatic nerve to mechanical stimulation may reflect two types of underlying genetic profiles. Such a possibility would be desirable for future studies.

Interestingly, ipsilateral side of the sciatic nerve, the one that received SNN surgery, all showed a similar profile of nerve firing, with a relatively consistent firing rate regardless of the strengths of the mechanical stimulation (Figure 3). The ipsilateral side of the sciatic nerve in monkey \#1 showed a near-basal level value at $1 \mathrm{~g}$ of stimulation force, before reaching the maximum firing rate. This suggested that the injured sciatic nerve was able to respond to stimulation. Thus, the constant firing rate for the SNN side of the sciatic nerve could be interpreted by a hypothesis of injury-resulted hyper-sensitization: trauma from the SNN injury caused the sciatic nerve to become hyper-sensitive, and normal level of input from the receptive field still led to maximum firing rate, therefore further stimulation would not be able to elicit any additional changes, thus making the SNN side of the sciatic nerve showing a "flat-lined" 
A

B
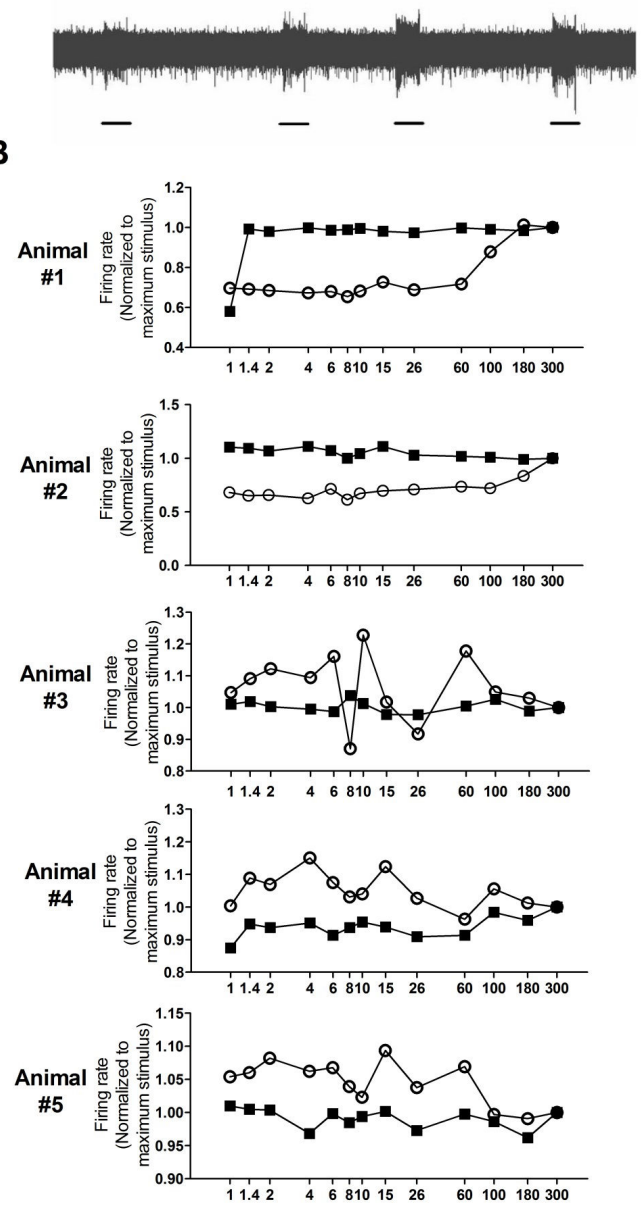

Stimulus Force (gram)

Figure 3: Sural nerve electrophysiological properties in monkeys with sciatic nerve neuropathy (SNN). Electrophysiological recordings were performed for a nerve bundle of the sural nerves of both the ipsilateral side and the contralateral side in five monkeys with SNN. The horizontal axis of each panel denotes the von Frey filament nominal force (in grams) that was applied to the receptive field of the nerve bundle being recorded. The vertical axis of each panel denotes the firing rate of the nerve bundle. Results for each nerve bundle were normalized to the data at the maximum stimulus. Open circles: recording data from the sural nerve of contralateral side. Closed squares: recording data from the sural nerve of ipsilateral side.

appearance of firing rate. Based on this hypothesis, the contralateral side of the sciatic nerve did not receive SNN injury, thus remaining in its native state of responsiveness, as demonstrated by changes in the firing rate profile in response to stimulation.

We adapted the nerve injury procedure of SNN based the chronic constriction injury model that first developed in rodents by Bennett and Xie [10], with suture ligatures tied around the sciatic nerve. In rodents, this procedure usually results in neuropathic pain, but without leg-guarding or usage avoidance, or prominent muscle atrophy. Why are there differences between those observed in rodents and those in monkeys? One possible contributing factor is species difference. There has been a reported case of tying ligature around sciatic nerve in monkey [19], and the authors noted that there was not the neuropathic pain behaviors in this monkey that were commonly observed in rodents. In an earlier study, monkeys received L5\&L6\&L7rhizotomy demonstrated paresis in affected legs and severe muscle atrophy in plantar extensors and dosiflexors, which the sciatic nerve innervates. The affected limbs were not in use and were passively dragged when the monkey moved about. In monkeys with less severe injury (L5- and L5\&L6-rhizotomy), the functions and the muscle volumes of the affected limbs were mostly retained. However, pain behaviors were not measured in these monkeys.

Conclusions

We constructed a model of sciatic nerve neuropathy (SNN) in the cynomolgus macaque monkey, Macaca fascicularis, and carried out initial characterization of the behavioral and physiological properties. SNN resulted in reduced leg usage and muscle atrophy. Sciatic nerve retained the ability of nerve signal transduction, and showed a flat-line type of firing rate profile consistent with the hypothesis of injuryresulted hyper-sensitization. These data suggest that mild injury to sciatic nerve result in long-lasting malfunction and neuropathy in monkeys. This model may serve as a non-human primate model to study functional changes, as well as underlying pathological mechanisms, of traumatic injury to the sciatic nerve.

\section{Acknowledgments}

We thank Mingjie He, Jihua Wang, Yang Wang and Liming Zheng for technical advice, and Hainan Jingang Laboratory Animal Co. Ltd. for providing technical assistance. This work was partially supported by funding from ShanghaiBio Corporation.

\section{References}

1. Saroyan JM, Winfree CJ, Schechter WS, Roye D, Gold AP (2007) Sciatic neuropathy after lower-extremity trauma: successful treatment of an uncommon pain and disability syndrome in an adolescent. Am J Phys Med Rehabil 86: 597-600.

2. Turan Ilica A, Yasar E, Tuba Sanal H, Duran C, Guvenc I (2008) Sciatic nerve compression due to femoral neck osteochondroma: MDCT and MR findings. Clin Rheumatol 27: 403-404.

3. Omezzine SJ, Zaara B, Ben Ali M, Abid F, Sassi N, et al. (2009) A rare cause of non discal sciatica: schwannoma of the sciatic nerve. Orthop Traumatol Surg Res 95: 543-546.

4. Eker HE, Cok OY, Aribogan A (2010) A treatment option for postinjection sciatic neuropathy: transsacral block with methylprednisolone. Pain Physician 13: 451-456.

5. Gauthier L, Allen D, Kim P (2010) Late transection of the sciatic nerve in association with an acetabular reconstruction cage. J Arthroplasty 25: 333.

6. Lohana P, Woodnutt DJ, Boyce DE (2010) Sciatic nerve palsy--a complication of posterior approach using enhanced soft tissue repair for total hip arthroplasty. J Plast Reconstr Aesthet Surg 63: e400-401.

7. Bistolfi A, Massazza G, Deledda D, Lioce E, Crova M (2011) Operative Management of Sciatic Nerve Palsy due to Impingement on the Metal Cage after Total Hip Revision: Case Report. Case Rep Med 2011: 830296.

8. Dubil EA, Dahle JM, Owens MD (2012) Bilateral sciatic nerve palsy: a new presentation of toilet bowl neuropathy. J Emerg Med 43: 622-624.

9. Wall PD, Devor M, Inbal R, Scadding JW, Schonfeld D, et al. (1979) Autotomy following peripheral nerve lesions: experimental anaesthesia dolorosa. Pain 7: 103-111. 
Citation: Guo N, Gu X, Xie Y, Zhao J, Xie Q, et al. (2014) Sciatic Nerve Neuropathy in Cynomolgus Monkey Macaca Fascicularis: Altered Leg Usage and Peripheral Nerve Firing. 2155-9562 5: 247. doi:10.4172/2155-9562.1000247

Page 6 of 6

10. Bennett GJ, Xie YK (1988) A peripheral mononeuropathy in rat that produces disorders of pain sensation like those seen in man. Pain 33: 87-107.

11. Sommer C, Petrausch S, Lindenlaub T, Toyka KV (1999) Neutralizing antibodies to interleukin 1-receptor reduce pain associated behavior in mice with experimental neuropathy. Neurosci Lett 270: 25-28.

12. Seltzer Z, Dubner R, Shir Y (1990) A novel behavioral model of neuropathic pain disorders produced in rats by partial sciatic nerve injury. Pain 43: 205-218.

13. Malmberg AB, Basbaum AI (1998) Partial sciatic nerve injury in the mouse as a model of neuropathic pain: behavioral and neuroanatomical correlates. Pain 76: 215-222.

14. Kim SH, Chung JM (1992) An experimental model for periphera neuropathy produced by segmental spinal nerve ligation in the rat. Pain 50: 355-363.

15. Kiso T, Watabiki T, Tsukamoto M, Okabe M, Kagami M, et al. (2008) Pharmacological characterization and gene expression profiling of an L5/L6 spinal nerve ligation model for neuropathic pain in mice. Neuroscience 153: 492-500.

16. Decosterd I, Woolf CJ (2000) Spared nerve injury: an animal model of persistent peripheral neuropathic pain. Pain 87: 149-158.

17. Bourquin AF, Süveges M, Pertin M, Gilliard N, Sardy S, et al. (2006) Assessment and analysis of mechanical allodynia-like behavior induced by spared nerve injury (SNI) in the mouse. Pain 122: 14 .
18. EDDS MV Jr, SMALL WT (1951) The behavior of residual axons in partially denervated muscles of the monkey. J Exp Med 93: 207-216.

19. Palecek J, Dougherty PM, Kim SH, Palecková V, Lekan H, et al. (1992) Responses of spinothalamic tract neurons to mechanical and thermal stimuli in an experimental model of peripheral neuropathy in primates. J Neurophysiol 68: 1951-1966.

20. Carlton SM, Lekan HA, Kim SH, Chung JM (1994) Behavioral manifestations of an experimental model for peripheral neuropathy produced by spinal nerve ligation in the primate. Pain 56: 155-166.

21. Landis SC, Amara SG, Asadullah K, Austin CP, Blumenstein R, et al. (2012) A call for transparent reporting to optimize the predictive value of preclinical research. Nature 490: 187-191.

22. Lapchak PA (2013) Recommendations and practices to optimize stroke therapy: developing effective translational research programs. Stroke 44: 841-843.

23. Ross KS, Marcri FJ (1975) The concentration of ascorbic acid in the posterior and anterior chambers of the rhesus monkey (Macaca mulatta). Invest Ophthalmol 14: 942-944.

24. Bito LZ, DeRousseau CJ, Kaufman PL, Bito JW (1982) Age-dependent loss of accommodative amplitude in rhesus monkeys: an animal model for presbyopia. Invest Ophthalmol Vis Sci 23: 23-31.

25. Thomas J, Carver M, Haisch C, Thomas F, Welch J, et al. (1982) Differential effects of intravenous anaesthetic agents on cell-mediated immunity in the Rhesus monkey. Clin Exp Immunol 47: 457-466. 